\title{
Carriage of multi-drug resistant bacteria among medical staff of the Yaoundé University Teaching Hospital, Cameroon
}

\author{
Gonsu K. H. ${ }^{1,3 \star}$, Ndongo G. A. ${ }^{1}$, Adiogo D. ${ }^{2}$, Toukam M. ${ }^{1,4}$, Ndze V. N. ${ }^{1}$, Kechia A. F. ${ }^{1}$ and Nkam \\ $M^{1,3}$ \\ ${ }^{1}$ Department of Microbiology, Hematology and Infectious Diseases, Faculty of Medicine and Biomedical Sciences, \\ University of Yaoundé 1, Cameroon. \\ ${ }^{2}$ Faculty of Medicine and Pharmaceutical Sciences, University of Douala, Cameroon. \\ ${ }^{3}$ Bacteriology Laboratory, Yaoundé University Teaching Hospital, Cameroon. \\ ${ }^{4}$ Clinical Biology Laboratory, General Hospital of Yaoundé, Cameroon.
}

Accepted 15 June, 2013

\begin{abstract}
Multidrug-resistant bacteria remain a serious worldwide public health problem. Health care workers are particularly implicated in dissemination of multidrug-resistant bacteria. The aim of this study was to determine the prevalence of multi-drug resistant bacteria (MRB) carriage by health care workers in high risk units of the Yaoundé University Teaching Hospital (YUTH). Nasal, rectal and hand swabs were collected from a sampled staff involved in patient care in the targeted units. The different bacteria were isolated on the appropriate selective culture media. The susceptibility of the different species isolated was done to confirm their different resistance phenotypes. Multidrug-resistant bacteria were isolated in $18.4 \%$ of the specimens and distributed in the different collection sites as follows: nose (60\%), rectum $(24 \%)$ and hands (16\%). Methicilin resistant Staphylococcus aureus represented $76 \%$ of these multidrug-resistant bacteria, extended spectrum beta-lactamase producing enterobacteracae (Klebsiella pneumoniae, Esherichia coli and Enterobacter cloacae) represented $22 \%$, and highly productive cephalorinase enterobacteracae (E. coli) represented $2 \% .62 \%$ of the staff included in the study carried at least a resistant bacterium. Health care worker in the Yaoundé University Teaching Hospital serve as an important reservoir for multidrug-resistant bacteria.
\end{abstract}

Key words: Multidrug-resistant bacteria, nosocomial infections, carriage, hospital staff.

\section{INTRODUCTION}

Resistant bacteria can lead to increased morbidity and mortality, especially in immunocompromised patients with serious underlying diseases. Antibiotic resistance poses problems both in the community and in health facilities. In hospitals, the transmission of bacteria is amplified because of the highly vulnerable patient population, and the selection pressure exerted by antibiotics. Bacteria are said to be multidrug resistant bacteria when there is an accumulation of natural and acquired resistance to com- monly used antibiotics of different families during active treatment (Guyot et al., 1999; Health Canada; 1998; CDC, 1998). Frequently encountered multidrug-resistant bacteria include Methicillin resistant Staphylococcus aureus (MRSA), Enterobacteraecae resistant to third generation cephalosporines (Escherichia coli, Enterobacter, Proteus), extended spectrum beta lactamase producing Enterobacteriaceae (ESBLE), cephalosporinases hyper productive Enterobacteriaceae (EBCASE), 
Pseudomonas aeruginosa resistant to ceftazidime (PARC), Acinetobacter baumannii resistance to ticarcillin and imipenem (ABRT) and vancomycin-resistant enterococci (VRE) (Health Canada, 1998; Lemaitre et al., 1996; Lee et al., 1998). They are involved in 20 to $30 \%$ of nosocomial infections (Lemaitre et al., 1996).

Morbidity attributable to healthcare associated infections is very important and affects hundreds and millions of people worldwide every year. At any time, over 1.4 million people worldwide suffer from this (Tikhomirov et al., 1987). All over the world, nosocomial infection is a recognized public health problem. Surveillance programmes estimated the rate of infection at 5 to $10 \%$ of hospital admissions (Pechere, 1993; Leroy, 1992). In developing countries, the risk of infection associated with healthcare is 2 to 20 times higher than in developed countries and the proportion of patients with nosocomial infection can exceed 25\% (Alliance Mondiale pour la sécurité des patients, 2005).

Resistance to antibiotics is associated with an increase in disease severity, which increases period of hospitalizetion, high mortality and increasing treatment costs, including a need for use of alternative drugs. The complex socioeconomic nature coupled with the misuse of antibiotics by health professionals, unskilled practitioners, layman, poor drug quality, unhygienic conditions for spread of resistant bacteria, and inadequate surveillance in developing countries can led to the selective spread of resistant organisms (Ogeer-Gyles, 2006).

The carriage of multidrug-resistant bacteria in colonized individuals or carriers represent a potential source of spread. The estimated number of patients with multidrugresistant bacteria on admission to any service stands at 25 to $40 \%$ (Niederman, 2001). Ignorance of asymptomatic carriage of multi-drug resistant bacteria (MRB) results in ignorance of a reservoir and therefore an increased risk of dissemination. Studies have demonstrated the role of caregivers in the chain of transmission of these infections with limited data on detection rates of multidrugresistant bacteria in health care workers in developing countries. Presently, no prevalence data on multidrug-resistant bacteria in healthcare workers in Cameroon exist.

It was therefore very important to determine the prevalence of multidrug-resistant bacteria and their antibiotic susceptibility pattern in potential reservoir of the medical staff, particularly in services that are at risk (intensive care, neonatology, surgical and obstetrical unit) in the Yaoundé University Teaching Hospital, Cameroon. This will probably improve the management of multidrug-resistant bacteria and also inform policy on the fight against nosocomial infection.

\section{METHODS}

\section{Study framework}

A descriptive study was carried out at the intensive care unit, neonatal care unit, surgical and obstetrical unit of the Yaoundé University Teaching Hospital, from May to August 2008. Included in the study were all medical, nursing, interns and surface staff in the services mentioned above. After obtaining the informed consent of the participant, four types of samples were collected from each participant using sterile swabs moistened with sterile saline. These included a nasal swab, a rectal swab, a left hand swab and a right hand swab. Samples were collected preferably in the middle of their shift periods.

\section{Culture and identification of MRB}

The different samples were inoculated on Trypticase soy agar (TSA) and also on appropriate selective media. For the isolation of MRSA, nasal and hand swabs were inoculated on Chapman agar (Biorad) supplemented with oxacillin at $2 \mathrm{mg} / \mathrm{l}$. Multidrug resistant Enterobacteriaceae, $P$. aeruginosa and $A$. baumannii were isolated on MacConkey + crystal violet (Biorad) agar supplemented with ceftazidime at $4 \mathrm{mg} / \mathrm{l}$ from rectal and hand swabs. Vancomycinresistant enterococci (VRE) were isolated on Columbia + ANC (Biorad) $+5 \%$ sheep blood supplemented with vancomycin at 6 $\mathrm{mg} / \mathrm{l}$. The samples were incubated at $37^{\circ} \mathrm{C}$ for 18 to $24 \mathrm{~h}$.

Presumptive identification was made after $24 \mathrm{~h}$ of incubation at $37^{\circ} \mathrm{C}$ by the cultural morphology, Gram stain, catalase and latex agglutination methods. All Gram negative bacteria isolates were identified by Gram staining, and by Analytical Profile Index (API) 20E and Analytical Profile Index (API) 20NE system (bioMerieux, Marcy l'Etoile, France). Pyruvate, tellurite and Pastorex Strepto Kit (Biorad) were used for the identification of enterococci.

\section{Susceptibility test and interpretation}

Antibiotic susceptibility was done by the Standardized Kirby-Bauer disc diffusion method with OSIRIS system (Biorad, Marne la Coquette, France) on Mueller-Hinton agar, as recommended by the Antibiogram Committee of the French Microbiology Society (ACFMS) for the characterization of ESBLE (CLIN Paris Nord, 1998). The quality control of discs used was performed using the following reference strains; Staphylococcus aureus (ATCC 25923), Escherichia coli (ATCC 25922) and P. aeruginosa (ATCC 27853). The resistance phenotypes particularly sought were: the resistance of $S$. aureus to oxacillin and cefoxitime for MRSA, ESBLE recognized by the synergy test between discs of third-generation cephalosporins, amoxicillin + clavulanic acid and susceptibility to cefoxitin (Pitout and Laupland, 2008), multi-drug resistant Enterobacteriaceae showing no synergy type "champagne cork" and resistant to cefoxitin were identified as EBCASE, $P$. aeruginosa resistant to ceftazidime (PARC) for their resistance to this antibiotic, the multidrug-resistant $A$. baumannii (ABRT) by resistance to ticarcillin and imipenem and vacomycin resistant enterococci (VRE) by their resistance to vancomycin. Phenotypic disc confirmatory test was performed as recommended by the Clinical and Laboratory Standards Institute (CLSI) (Clinical and Laboratory Standards Institute, 2009). It is also important to note that molecular methods to confirm different bacteria phenotypes and minimum inhibitory concentrations (MICs) of antibiotics used were not determined because of limited resources. The identified multidrug-resistant bacteria (MRB) were stored in Trypticase soy (TS) broth glycerol at $-20^{\circ} \mathrm{C}$.

\section{Data analysis}

Data was analyzed using Microsoft excel and SAS software v8.2. Prevalence was expressed as percentages. The Chi square $\left(X^{2}\right)$ test was used to analyze categorical variables and the Yates correction test where appropriate. A 95\% confidence interval $(\mathrm{Cl})$ was calculated and values of $P<0.05$ were considered significant. 
Table 1. Percentage of the different multidrug-resistant phenotypes isolated.

\begin{tabular}{llcccc}
\hline MRB & & Number & MRB (50) (\%) & Staff collected (66) (\%) & Samples collected (264) (\%) \\
\hline MRSA & & 38 & 76 & 53 & 19.5 \\
& K. pneumoniae & 6 & 12 & 9.1 & 3.0 \\
EBLSE & E. coli & 4 & 8 & 4.5 & 1.5 \\
& E. cloacae & 1 & 2 & 1.52 & 0.5 \\
EBCASE & E. coli & 1 & 2 & 1.52 & 0.5 \\
Total & & 50 & 100 & 74.2 & 25.0 \\
\hline
\end{tabular}

\section{Ethical issues}

An ethical clearance was obtained from the Cameroon National Ethics Committee, informed consent obtained from the participants and an administrative authorization was obtained from the Yaoundé University Teaching Hospital.

\section{RESULTS}

A total of 66 participants were recruited in this study. This study showed that the intensive care participants represented $54.5 \%$ of the sample size followed by the neonatology unit $(19.70 \%)$, the surgical block $(13.6 \%)$ and obstetrical block (12.1\%). A total of 264 different samples were collected in this study. Regarding the category of staff, nursing interns and nurses represented respective collection rates of 92.3 and $69.8 \%$. The cleaning staff follows with a collection rate of $55.5 \%$ while collection rate among doctors was only $20 \%$. The ages of the staff vary between 21 and 54 years with a mean \pm SD age of $37 \pm 22$ years. The participation of female staff was higher $(62 \%)$ than male $(38 \%)$ with a sex ratio (male: female) of 0.65 .

Multidrug-resistant bacteria were identified in 50 (18.9\%) samples. Out of the total MRB isolated, $60 \%$ were isolated from the nose, $24 \%$ from the rectum and $16 \%$ from hands.

Three phenotypes of MRB were identified: Methicillin resistant S. aureus (MRSA) constituted $76 \%$ of MRB, Extended spectrum beta lactamase producing Enterobacteriaceae (ESBLE) constituted $22 \%$ with the species Klebsiella pneumoniae, Enterobacter cloacae and E. coli isolated and $2 \%$ of cephalosporinases hyper productive Enterobacteriaceae (EBCASE) with E. coli

being the only species isolated in this group (Table 1).

The study of the sensitivity profile of the 50 strains to antibiotics showed that all strains were multidrug-resistant and was of help to identify the different resistance phenotypes. The resistance of MRSA to beta-lactams was characteristic but other associated resistance phenotypes were observed, including a high resistance to aminoglycosides and pefloxacine. The lincomicine, pristinamycin and minocycline were however still active. With regard to the glycopeptides, teicoplanin resistance of the tested strains was 73.7 and $31.6 \%$ for vancomycin. All MRSA strains isolated were resistant to trimethoprim + sulfamethoxazole (Figure 1).
Eleven (11) strains of extended spectrum betalactamase producing Enterobacteriaceae were isolated from these samples. They represented a $22 \%$ rate of isolated MRB and $5.5 \%$ of the samples where they were investigated. Regarding the associated resistances, the main phenotypes of resistance to aminoglycosides were observed for these strains. Cross resistance to fluoroquinolones were also observed (54.5\% of strains resistant to at least one quinolone). All strains were also resistant to trimethoprim + sulfamethoxazole. Imipenem remained active for all these strains (Figure 2).

The single EBCASE strain isolated from a rectal swab was $E$. coli. This strain did not show synergy between discs of third-generation cephalosporins and amoxicillin + clavulanic acid. This strain was resistant to all antibiotics tested with the exception of imipenem and amikacin.

The distribution of MRB by service showed that the intensive care unit had a rate of $60 \%$ of staff carrying MRB and constituted $36.4 \%$ of all MRB isolated. In contrast, carriage rates of MRB as compared to the size of each target service showed fairly similar rates for all services (Table 2). Table 3 shows the distribution of MRB by staff category. It was observed that working in a particular hospital service did not influence the carriage of MRB by personnel, $X^{2}$ (Yates) $=0.030, P=0.86$.

\section{DISCUSSION}

This survey is the first of its kind on the medical staff in Cameroon. The choice of the intensive care units, neonatal, surgical and obstetrical blocks as target services was guided by the recurrent appearance of multidrugresistant $K$. pneumoniae infections in these units at the Yaoundé University Teaching Hospital. MRB were found more frequently in the nasal cavity $(60 \%)$. This is higher than that obtained by Gualdoni and co-worker in Vienna (Gualdoni et al., 2012). The rectal site follows with a percentage of $24 \%$ and hands with $16 \%$ of MRB. In general, nasal carriage rates among hospital personnel and patient $(60$ to $70 \%)$ are much higher than those in community carriers (30 to 50\%) (Lowy, 1998). In the present study, $76 \%$ of MRB were MRSA and this is much higher than that obtained in other studies (Farzana et al., 2008; Akoua et al., 2004). According to Lepori (2006), 80\% of hand samples carry MRSA (Lepori, 2006), meanwhile 


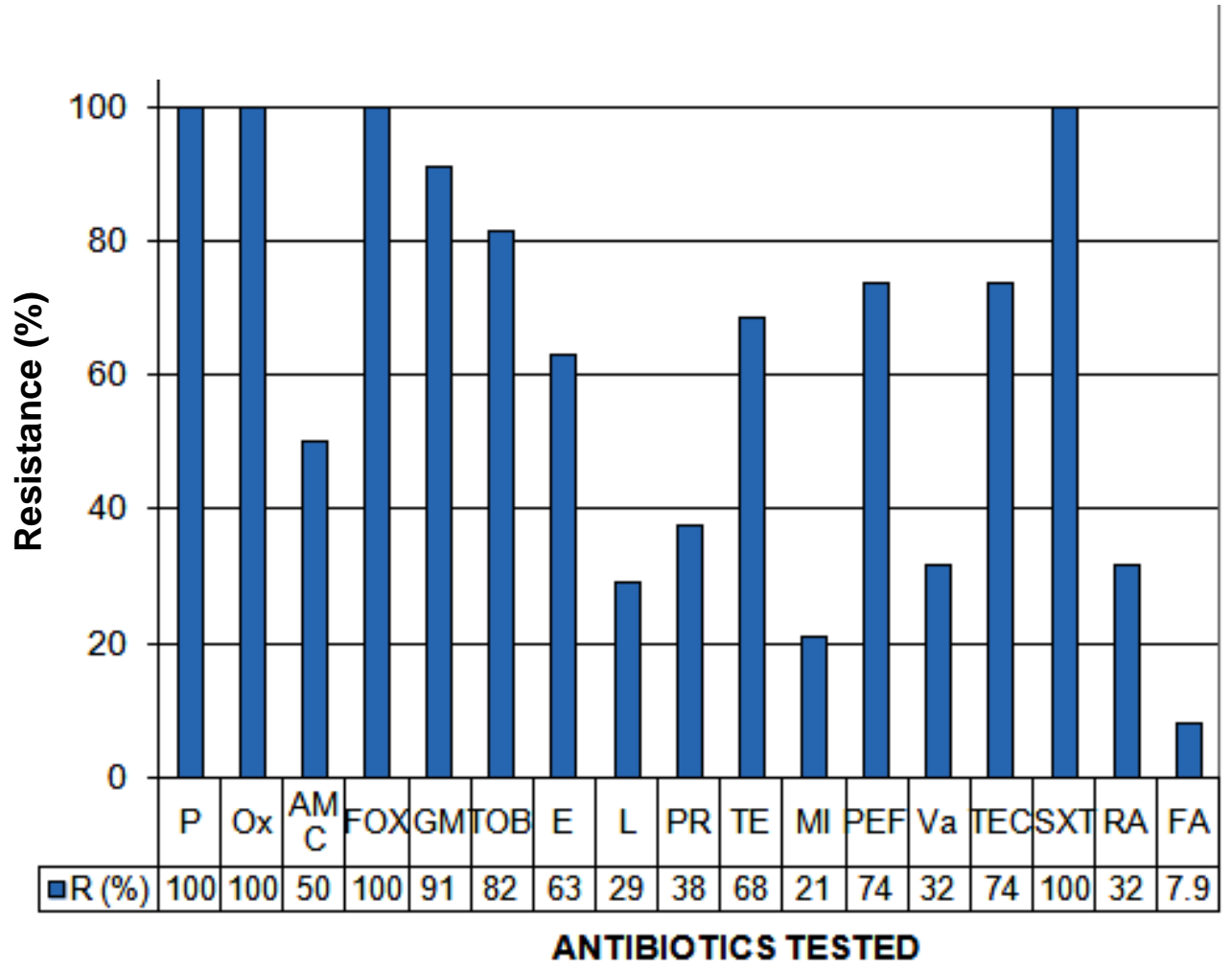

P-Penicillin

ox-Axacillin

AMC-Amoxicillin

FoX-Cefoxitine

GM-Gentamicin

TOB-Tobramycin

E- Erythromycine

L- Lincomycin

PR- Pristinamycin

TE-Tetracycline

MI-Minocycline

PEF-Perfloxacine

Va-Vancomycin

TEC-Teicoplanin

SXT-

Trimothroprim+sulf.

amethaxole.

RA-Rifamicin

FA- Fusidic acid

Figure 1. Antibiotic resistance profile of the $38 \mathrm{MRSA}$ isolated.

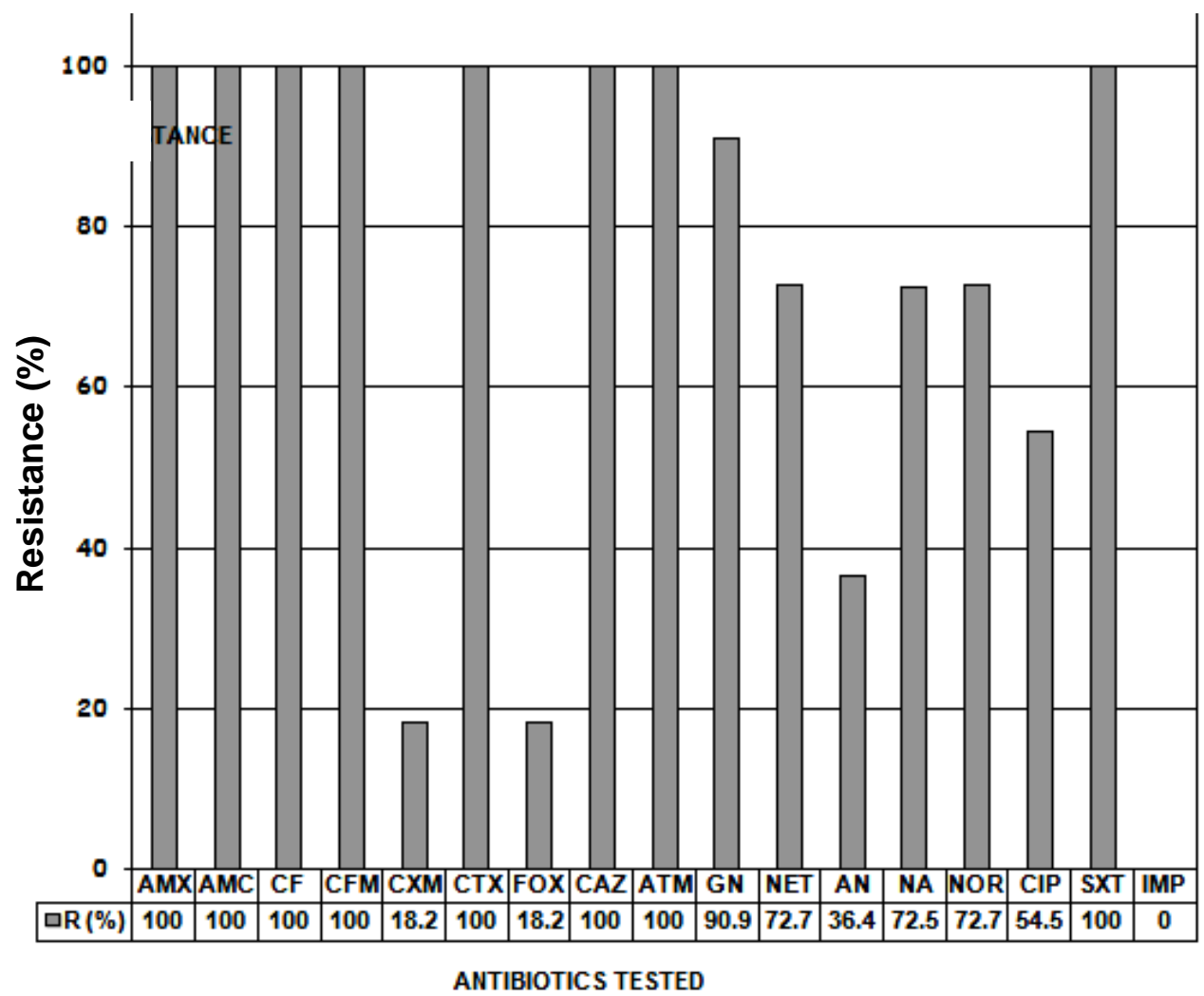

AMX-amoxicillin

AMC-amoxicillin+clavunillic

acid

CF-cefalotine

CFM-cefixime

CXM-cefuroxime

CTX-cefotaxime

FoX-cefoxitine

CAZ-ceftazidime

ATM-aztreonam

GN-gentamicin

NET-netilmicine

AN-amikacin

NA-nalidixiçacid

NOR-norfloxacine

CIP- ciprofloxacine

SXT-

trimethoprime+sulfamethoxaz

ole

IMP-imipinem

Figure 2. Antibiotic resistance profile of 11 strains of ESBLE isolated. 
Table 2. Distribution of MRB by hospital unit.

\begin{tabular}{lcccc}
\hline MRB & Intensive care & Neonatology & Surgical block & Obstetrical block \\
\hline Number & 24 & 8 & 5 & 5 \\
MRB (\%) & 36.4 & 9.1 & 7.6 & 7.6 \\
Staff no. (\%) & 66.7 & 46.1 & 55.6 & 62.5 \\
Total no. (\%) & 60.0 & 15.0 & 12.5 & 12.5 \\
\hline
\end{tabular}

Table 3. Distribution of MRB by staff category.

\begin{tabular}{lcccc}
\hline MRB & Doctors & Nurses & Cleaning staff & Interns \\
\hline Number & 2 & 27 & 4 & 7 \\
MRB (\%) & 3.0 & 40.9 & 6.0 & 10.6 \\
Staff category no. (\%) & 40.0 & 62.8 & 80.0 & 53.8 \\
Total no. (\%) & 5.4 & 67.5 & 10.0 & 17.5 \\
\hline
\end{tabular}

only $26.6 \%$ of hand samples carried MRSA in this study. The rate of MRB obtained at the hand samples could be biased by the fact that some staff had washed their hands prior to sampling. The presence of MRB in the hands even evokes the possibility of hand transmission to patients or other health care workers and indirectly to the environment.

Extended spectrum beta lactamase producing Enterobacteriaceae (ESBLE) constituted 22\%. This is lower than that obtained by Yusuf et al. (2011) in Kano. This is quite higher when compared with the findings of Aibinu and co-workers (2003) who reported $20 \%$ prevalence in Lagos-Nigeria, $4.8 \%$ in Saudi Arabia (Kader and Kumar, 2005) and a prevalence rate of $12 \%$ in Yaoundé, Cameroun (Gangoue-Pieboji et al., 2005; El-Khizzi and Bakheshwain, 2006). The ESBLE and EBCASE were isolated from the rectal swab (24\%).

The results obtained in this study on the distribution of MRB isolated from the medical staff by service (Table 2) shows however that the percentage of MRB carriage was similar: $66.7 \%$ in intensive care, $46.1 \%$ in neonatal care, $55.6 \%$ in surgical block and $62.5 \%$ in the obstetric unit. This could be explained by the fact that all the services were chosen based on the fact that they are high risk units for MRB compounded by the selective pressure of antibiotics even if that risk is not perceived the same way by the nursing staff. These are also services where patients are most dependent and "manipulated" by the nursing staff.

The nursing personnel were most affected by the carriage of multidrug-resistant bacteria $(40.9 \%$ as compared to $67.5 \%$ MRB isolated from the total staff) as shown in Table 3. This could be explained by the fact that nurses are more in contact with patients and they practice more repetitive gestures, sometimes invasive on patients. The cleaning staff appeared with a percentage of $80 \%$ as compared to the numbers collected in this category. The staff was particularly involved in surface cleaning and waste disposal at YUTH, which would explain this high rate. However, these results should be interpreted with caution because the number of such staff, as well as that of doctors was very low (5 staff per category) and certainly not sufficiently representative. We would have expected to see less experienced staff intern more affected than the older and regular staff. Nonetheless, being a "learner" nurse did not expose them more to MRB.

\section{Conclusion}

This work confirms a high carriage rate of MRB organisms by healthcare staff. The associated phenotypic resistances observed in this study require that warning be made to the services in which this was observed. Strict compliance to "standard" precautions by all and for all is the basic measure to prevent cross-transmission of MRB.

\section{ACKNOWLEDGEMENTS}

We thank $\mathrm{Dr}$ Michel Ndoumbe Nkeng for statistical analysis and the Yaoundé University Teaching Hospital for providing laboratory facilities and technical guidance for successful completion of this work. We also wish to thank the staff of the hospital who participated in this study.

\section{REFERENCES}

Aibinu I, Odugbemi P, Brian JM (2003). Extended-spectrum $\beta$ lactamase in isolates of Klebsiella spp and Escherichia coli from Lagos. Nig. J. Health Biomed. Sci. 2:53-60.

Akoua KC, Toure R, Guessennd N, Acho B, Faye K, Loukou YG, Dosso M (2004). Nasal carriage of meticillin-resistant Staphylococcus aureus among health care personnel in Abidjan. Dakar. Med. 49:7074

Alliance Mondiale pour la sécurité des patients (2005). Recommandations OMS pour la L'Hygiène des mains au cours des soins 
(version avancée).Synthèse. "Des mains propres sont des mains plus sures ». p.31.

CDC guidelines for infection control in hospital personnel. (1998). Am. J. Infect. Contr. 26:289-354.

CLIN Paris Nord (1998). Fiches de recommandation: Maîtrise de la diffusion des bactéries multi résistantes aux antibiotiques; 44:6-13.

Clinical and Laboratory Standards Institute (2009). Methods for Dilution Antimicrobial Susceptibility Tests for Bacteria that Grow Aerobically; Approved Standard. 8th ed. Pennsylvania, USA: Clinical and Laboratory Standards Institute; (CSLI document; M07-A8). ISBN 156238-689-1.

El-Khizzi NA, Bakeshwain SM (2006). Prevalence of extended spectrum betalactamases among Enterobacteriacea isolated from blood culture in a tertiary care hospital. Saudi Med. J. 27(1):37-40.

Farzana K, Rashid Z, Akhtar N, Sattar A, Khan JA, Nasir B (2008). Nasal carriage of Staphylococci in health care workers: antimicrobial susceptibility profile. Pak. J. Pharm. Sci. 21(3):290-294.

Gangoue-Pieboji J, Benedic B, Koulla-Shiro S, Randegger C, Adiogo D, Ngassam $P$, Ndumbe $P$, Hachler H (2005). ESBL producing Enterobacteriacea in Yaounde, Cameroon. J. Clin. Microbiol. 43(7):3237-3247.

Gualdoni GA, Lingscheid T, Tobudic S, Burgmann H (2012). Low nasal carriage of drug-resistant bacteria among medical students in Vienna. GMS Krankenhaushygiene Interdisziplinär., 7:1.

Guyot A, Barrett SP, Threlfall EJ, Hampton MD, Cheasty T (1999). Molecular epidemiology of multi-resistant Escherichia coli. J. Hosp. Infect. 43:39-48.

Health Canada (1998). Hand washing, cleaning, disinfection, and sterilization in health care. Canada Communicable Disease Report (CCDR), 24(8):13.

Lee TB, Baker OG, Lee JT, Scheckler WE, Steele L, Laxton CE (1998). Recommended practices for surveillance. Am. J. Infect. Contr. 26:277-288.
Lemaitre N, Jarlier V (1996). Dépistage des porteurs de klebsielles productrices de bêtalactamases à spectre étendu à l'entrée des unités de réanimation: problèmes techniques et rendement. In: Grosset J, Kitzis M, Lambert N, Sinegre M, éds. Prévention contre les germes multirésistants. Paris: Arnette; pp.105-108.

Lepori ML (2006). BMR et personnel soignant, Journées ANMTPH Marseille.

Leroy E (1992). Denying microorganisms their chance. Afr. Health 14:37-38

Lowy FD (1998). Staphylococcus aureus infections. N. Engl. J. Med. 339:520-532.

Niederman MS (2001). Impact of antibiotic resistance on clinical outcomes and the cost of care. Crit. Care Med. 29:114-120.

Ogeer-Gyles JS (2006). Nosocomial infections and antimicrobial resistance in critical care medicines. J. Vet. Emerg. Crit. Care. 16(1):1-18.

Pechere JC (1993). Microbiology of nosocomial infections. Bull Acad Natl Med. 177:705-715.

Pitout JDD, Laupland KB (2008). Extended-spectrum beta-lactamase producing Enterobacteriaceae: an emerging public-health concern. Lancet Infect. Dis. 8:159-166.

Tikhomirov E (1987). WHO Programme for the Control of Hospital Infections. Chemiotherapia, 3:148-151.

Yusuf I, Arzai AH, Umar A, Magaji N, Salisu N, Tukur A, Haruna M, Hamid KM (2011). Prevalence of Extended Spectrum Beta Lactamases (ESBL) producing Escherichia coli and Klebsiella Pneumoniae in Tuberculosis patients $\mathrm{n}$ Kano, Nigeria. Bajopas., 4(2):182-185. 